\title{
Erratum to: A Case Series and Literature Review of Merkel Cell Carcinoma Metastasizing to Pancreas
}

\author{
Yezaz A. Ghouri ${ }^{1,2}$ - Somashekar G. Krishna ${ }^{3}$ Uma R. Kundu ${ }^{4} \cdot$ Manoop S. Bhutani $^{1}$ • \\ Jeffrey H. Lee ${ }^{1} \cdot$ William A. Ross ${ }^{1}$
}

Published online: 15 May 2015

(C) Springer Science+Business Media New York 2015

\section{Erratum to: Dig Dis Sci \\ DOI 10.1007/s10620-014-3516-5}

In the original publication, the fourth coauthor's surname was incorrectly published as Butani. The correct surname should be Bhutani.

The online version of the original article can be found under doi:10.1007/s10620-014-3516-5.

William A. Ross

wross@mdanderson.org

Yezaz A. Ghouri

yezaz.a.ghouri@uth.tmc.edu

Somashekar G. Krishna

somashekar.krishna@osumc.edu

Uma R. Kundu

urkundu@mdanderson.org

Manoop S. Bhutani

manoop.bhutani@mdanderson.org

Jeffrey H. Lee

jefflee@mdanderson.org
1 Division of Gastroenterology, Hepatology and Nutrition, University of Texas-MD Anderson Cancer Center, 1515 Holcombe Boulevard, Unit 1466, Houston, TX 77030, USA

2 Department of Internal Medicine, University of Texas Health Science Center at Houston, 6431 Fannin Street, MSB 1.134, Houston, TX 77030, USA

3 Department of Gastroenterology, Hepatology and Nutrition, Ohio State University Wexner Medical Center, 395 W. Doan, Suite 200, Columbus, OH 43210, USA

4 Department of Pathology, University of Texas-MD Anderson Cancer Center, 1515 Holcombe Boulevard, Unit 0085, Houston, TX 77030, USA 